\title{
Endovascular Management of a Rare Cause of Bleeding Per Vaginam: A Hole in the Mole
}

Anurag Chahal ${ }^{1} \quad$ Sundeep Malla ${ }^{1} \quad$ Shivanand Gamanagatti

\begin{abstract}
Address for correspondence Shivanand Gamanagatti, MD, Department of Radiodiagnosis, All India Institute of Medical Sciences, New Delhi 110029, Delhi, India (e-mail: shiv223@gmail.com).
\end{abstract}

\begin{abstract}
Keywords

- uterine artery

- pseudoaneurysm

- embolization

Uterine artery aneurysm is a rare cause of bleeding per vaginam. Uterine artery pseudoaneurysms are rare but potentially life-threatening causes of persistent or delayed bleeding post-uterine curettage or any uterine surgery. Color Doppler ultrasonography is very useful for emergent diagnosis of these conditions and subsequent follow-up post-embolization. Computed tomography scan and magnetic resonance imaging play an important role in confirming the diagnosis. Transcatheter arterial embolization is the therapy of choice for such cases. Our case highlights the presentation, imaging appearance, and endovascular management of a case of uterine artery pseudoaneurysm.
\end{abstract}

\section{Introduction}

Uterine artery aneurysm is a rare cause of bleeding per vaginam. It is usually caused by traumatic injury to the vessels during cesarean section, myomectomy, or post-dilatation and curettage. The patient usually presents with bleeding per vaginam that characteristically occurs after some delay post the inciting event. A timely screening with ultrasound and further workup with magnetic resonance imaging (MRI) can help in timely diagnosis of this potentially life-threatening entity. Transcatheter uterine artery embolization is an effective and minimally invasive treatment option in patients who wish to preserve their reproductive potential.

\section{Case Report}

\section{Presentation}

A 24-year-old woman presented to our emergency department with heavy bleeding per vaginam for the past 15 days. On examination, the patient had severe pallor, blood pressure of $96 / 62 \mathrm{~mm} \mathrm{Hg}$, and a pulse rate of 102 beats/min. Hemoglobin level was $6.4 \mathrm{~g} / \mathrm{dL}$. She had a history of suction evacuation 6 months back for molar pregnancy, followed by chemotherapy owing to her high World Health Organization (WHO) risk score. Serum $\beta$-human chorionic gonadotropin $(\beta-\mathrm{HCG})$ was undetectable.

A pelvic ultrasound showed a small anechoic lesion in

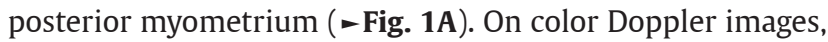
there was presence of color flow with alternating red and

received

November 19, 2018

accepted after revision

January 28, 2019

published online

April 18, 2019 blue colors with in the lesion ( $\mathbf{- F i g . ~ 1 B ) , ~ w h i c h ~ i s ~ d i a g n o s t i c ~}$ of a pseudoaneurysm (yin-yang sign). Because the patient was hemodynamically stable and not actively bleeding at the time of presentation, an MRI of the pelvis was done for better characterization of the lesion. MRI ( - Fig. 2) showed a large lesion with central T2 dark flow void in posterior myometrium, which showed intense enhancement in the arterial phase with a peripheral T1/T2 hyperintense lesion without contrast enhancement. Thus a diagnosis of pseudoaneurysm with surrounding nonenhancing hematoma without any evidence of gestational trophoblastic neoplasm (GTN) was made.

\section{Treatment}

The patient was taken for transcatheter embolization after written informed consent that she might have suboptimal fertility if permanent proximal bilateral uterine artery embolization was deemed necessary. After taking access through right common femoral artery, pelvic angiography ( - Fig. 3A) was performed, followed by selective left internal iliac ( - Fig. 3B) and left uterine artery angiography ( - Fig. 3C) using Robertson uterine catheter (RUC). A selective run of the uterine artery was taken using 2.7F microcatheter (Progreat, Terumo Corporation), and care was taken to position the microcatheter distal to any branch to prevent nontarget embolization. Because the supplying vessel was very tortuous and long with a large stump leading to pseudoaneurysm, formulation of $\mathrm{N}$-butyl cyanoacrylate (NBCA) glue was 

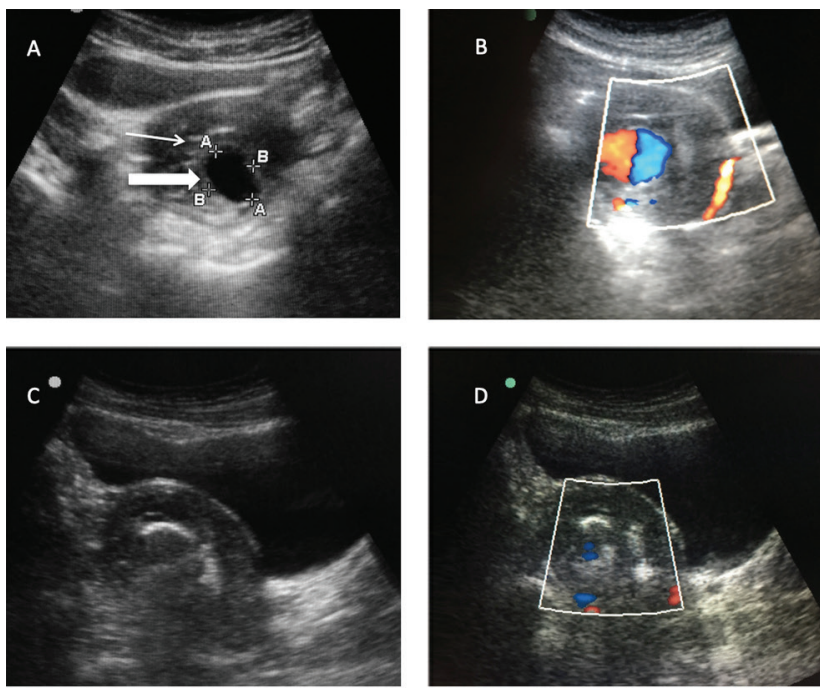

Fig. 1 Longitudinal image of USG pelvis (A) shows an enlarged uterus with an anechoic, well-defined round lesion (block arrow) in posterior myometrium, displacing the echogenic endometrial stripe (arrow) anteriorly. On color Doppler (B), it shows a typical yinyang color flow pattern with alternating color flow within the sac. Post-embolization ultrasound image (C) shows echogenic contents within the previously anechoic lesion. Color flow image (D) does not show any significant color flow within the sac s/o complete thrombosis of the pseudoaneurysm.

diluted (20\%) with Lipiodol to prevent proximal occlusion. We avoided gel foam embolization of contralateral side because she was a nulliparous young woman and any decrease in her fertility is not warranted as a consequence of the procedure. Post-embolization run shows cutoff of arterial branch leading to the pseudoaneurysm with glue cast forming just proximally ( - Fig. 3E). A contralateral run showed enlarged right uterine artery but no supply to the pseudo aneurysm ( - Fig. 3F). Follow-up ultrasound showed echogenic contents within the previously anechoic cavity (hole) ( - Fig. 1C) and no color flow within the lesion ( - Fig. 1D).

\section{Follow-up}

Within a few hours of procedure, bleeding per vaginam stopped. The patient was hemodynamically stable and was discharged the next day. She was followed up up to 1 year, and no evidence of recurrence was seen. She had conceived and was in her 32nd week of gestation at the time of submitting this manuscript.

\section{Discussion}

Gestational trophoblastic disease (GTD) is a condition characterized by abnormal proliferation of trophoblastic tissue. ${ }^{1}$ Suction evacuation and curettage, which is a common therapeutic measure for GTD, can cause uterine vascular abnormalities, including acquired arteriovenous malformations (AVMs), pseudoaneurysms, arteriovenous fistulas, and even rupture of vessels. Recognition of these abnormalities as the cause of delayed hemorrhage is important, because these abnormalities can be treated effectively and safely by endovascular transcatheter embolization.
Pseudoaneurysms are formed due to inadequate sealing of any injury to the arterial wall during surgery or penetrating trauma. Blood extravasates into the surrounding tissues under pressure forming a sac that is communicating with the arterial lumen. Curettage and cesarean section are the common causes of uterine artery pseudoaneurysms. ${ }^{2}$ A pseudoaneurysm may be asymptomatic, may rupture, or get thrombosed with resulting distal embolization. The risk of rupture correlates directly with the intramural pressure and pseudoaneurysm size. Urgent intervention is required on detection, as pseudoaneurysms are at risk of rupturing or expanding that can be catastrophic. ${ }^{3}$ Uterine artery pseudoaneurysms or AVM should always be suspected in cases of recurrent or persistent bleeding per vaginam after GTN.

Acute treatment consists of hemodynamic stabilization and control of active bleeding. Occlusion with a Foley catheter bulb may be effective. Ultrasound with Doppler study is the first and probably the only investigation in an emergent setting in such cases owing to its high sensitivity to pick up vascular causes of bleeding. Uterine pseudoaneurysms appear as an anechoic round lesion (hole!) within the myometrium or endometrium along with swirling arterial flow with different directions and velocities within the sac. ${ }^{4}$ It is important to assert that MRI is only validated in nonemergent cases in which it is worthwhile to look for any residual GTN along with the aneurysm, which could be a cause of recurrence of the pseudoaneurysm after embolization and would warrant a closer watch on $\beta$-HCG levels.

Endovascular uterine artery embolization is the ideal therapy in patients who wish to retain reproductive capacity owing to excellent success rates and low complication rate. ${ }^{5}$ In case of proximal or large feeders of pseudoaneurysm or arteriovenous fistula, metallic coils are preferred embolizing agents to avoid crossover to venous side. NBCA glue or gel foam can be used for pseudoaneurysms without an underlying arteriovenous fistula. ${ }^{6} \mathrm{Gel}$ foam is good due to its temporary nature, and thus it produces good fertility outcomes but carries a risk of recanalization, especially in the setting of a GTN. NBCA, on the other hand, is permanent embolizing agent and requires a good expertise due to its propensity for nontarget embolization. ${ }^{6}$ Transvaginal ultrasound-guided thrombin injection has also been used in a few cases. ${ }^{7} \mathrm{~A}$ post-embolization contralateral check run was performed, and the patient is kept on follow-up with serial $\beta$-HCG titers.

\section{Conclusion}

Uterine artery pseudoaneurysms are rare but potentially life-threatening entities. Color Doppler ultrasonography is an ideal modality for emergent diagnosis of these conditions and follow-up of patients after embolization. Transcatheter arterial embolization is the therapy of choice for these iatrogenic vascular abnormalities.

\section{Informed Consent Statement}

Informed consent was obtained from the patient. 

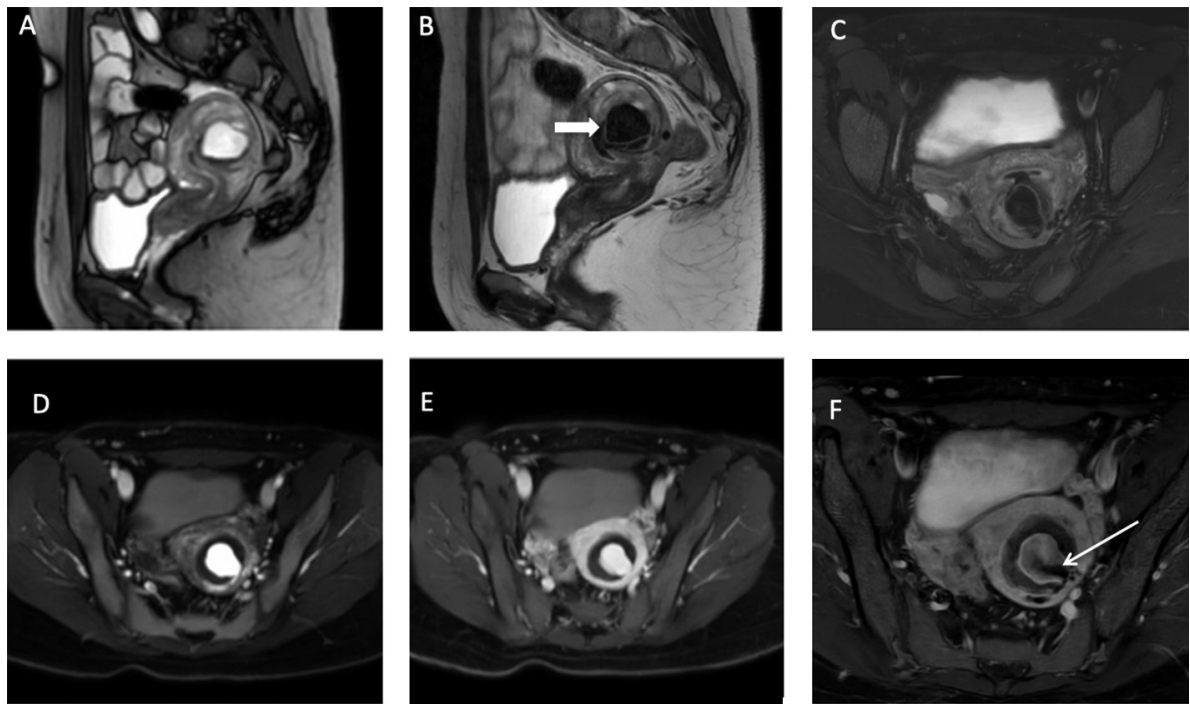

Fig. 2 MRI of pelvis shows a round lesion in posterior myometrium that is bright on gradient images (A) and shows a signal void on T2 images (B, C). This lesion shows intense homogeneous enhancement in arterial phase of postcontrast images (D), which persisted in venous phase images (E) suggestive of pseudoaneurysm. (F) Also, a delayed postcontrast image shows a persistent contrast enhancement with a black jet (arrow) seen to project into the cavity likely to be the neck of the pseudoaneurysm. There is a T2 heterogeneous area (block arrow in B) with no enhancement on postcontrast images surrounding the pseudoaneurysm s/o thrombus.
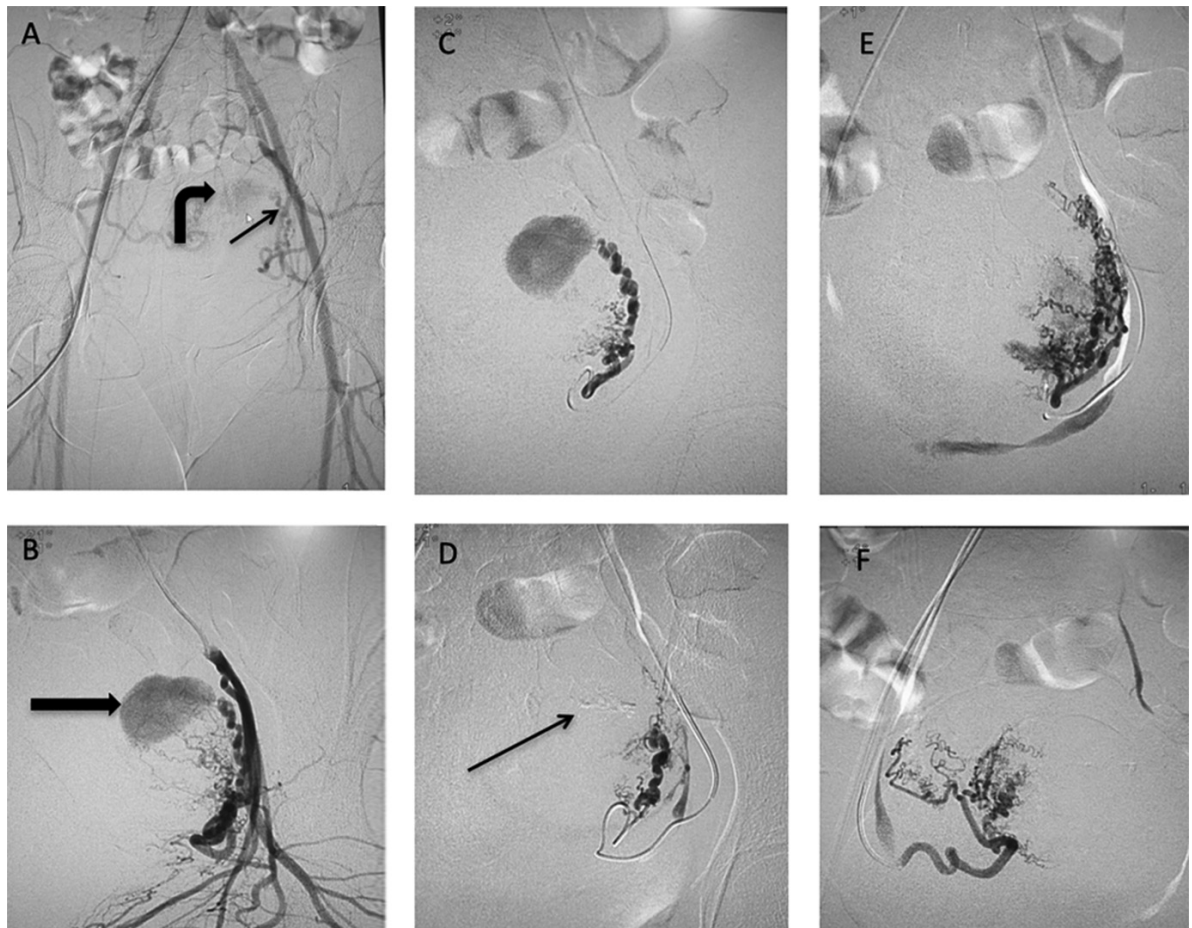

Fig. 3 A pelvic angiogram (A) is done keeping a catheter in infrarenal aorta with faint opacification of left uterine artery (straight arrow) and a contrast filled sac (curved arrow) from distal end of left uterine artery. After selective catheterization of left Internal iliac artery, an angiogram (B) shows enlarged tortuous left uterine artery and a large pseudoaneurysm (block arrow) arising from it. Further superselective catheterization of left uterine artery was done using a microcatheter, and angiogram (C) shows better opacification of uterine artery and pseudoaneurysm. After negotiating the cervicovaginal branch, $20 \%$ glue embolization was done. Post-embolization images (D) show a glue cast (arrow) in left distal uterine artery just proximal to pseudoaneurysm. A repeat post-embolization run after withdrawing the microcatheter (E) shows complete cutoff of pseudoaneurysmal sac. Right uterine artery run (F) shows no filling of pseudoaneurysm or any other vascular abnormality.

\section{Note}

Our case report highlights a rare case of uterine artery pseudoaneurysm as a cause of bleeding per vaginam, with the role of interventional radiology in its management. Interventional radiology is playing an immaculate role in the management of uterine artery pseudoaneurysms that otherwise pose a grave challenge to the gynecologist. 


\section{Ethical Approval Statement}

All procedures performed in studies involving human participants were in accordance with the ethical standards of the institutional ethics committee and with the 1964 Helsinki declaration and its later amendments or comparable ethical standards.

\section{Conflict of Interest}

None.

\section{References}

1 Biscaro A, Braga A, Berkowitz RS. Diagnosis, classification and treatment of gestational trophoblastic neoplasia. Rev Bras Ginecol Obstet 2015;37(1):42-51

2 Zimon AE, Hwang JK, Principe DL, Bahado-Singh RO. Pseudoaneurysm of the uterine artery. Obstet Gynecol 1999;94(5 Pt 2):827-830
3 Karmous N, Ayachi A, Derouich S, Mkaouar L, Mourali M. Rupture of uterine artery pseudoaneurysm: role of ultrasonography in postpartum hemorrhage management. Pan Afr Med J 2016;25:136

4 Langer JE, Cope C. Ultrasonographic diagnosis of uterine artery pseudoaneurysm after hysterectomy. J Ultrasound Med 1999;18(10):711-714

5 Keepanasseril A, Suri V, Prasad GRV, et al. Management of massive hemorrhage in patients with gestational trophoblastic neoplasia by angiographic embolization: a safer alternative. J Reprod Med 2011;56(5-6):235-240

6 Kim GM, Yoon CJ, Seong NJ, Kang S-G, Kim Y-J. Postpartum haemorrhage from ruptured pseudoaneurysm: efficacy of transcatheter arterial embolisation using N-butyl-2-cyanoacrylate. Eur Radiol 2013;23(8):2344-2349

7 Ros C, Barnes D, Fervienza A, et al. Ultrasound-guided transvaginal thrombin injection of uterine arteries pseudoaneurysms. Br J Radiol 2017;90(1074):20160913 